\begin{tabular}{|l|l|}
\hline Postprint Version & 1.0 \\
\hline Journal website & http://www.pec-journal.com/article/S0738-3991\%2810\%2900326-5/abstract \\
\hline Pubmed link & http://www.ncbi.nlm.nih.gov/pubmed/20638817 \\
\hline DOI & $10.1016 / j . p e c .2010 .05 .033$ \\
\hline
\end{tabular}

This is a NIVEL certified Post Print, more info at http://www.nivel.eu

\title{
The silent healer: The role of communication in placebo effects
}

\author{
JOZIEN M. BENSING ${ }^{\text {A },{ }^{\mathrm{B}}, * \text {, WILLIAM VERHEUL }}{ }^{\mathrm{A}}$ \\ ${ }^{a}$ NIVEL (Netherlands Institute for Health Services Research), The Netherlands \\ ${ }^{b}$ Faculty of Social and Behavioural Science, Utrecht University, The Netherlands
}

\begin{abstract}
Placebo effects have an ambiguous reputation, as they are associated with sham treatment and deceit on the one hand and as interesting phenomena, which might be clinically relevant on the other. The goal of this paper is to demonstrate that placebo effects are relevant and can be used as an effective part of many treatments by using communication targeting placebo effect mechanisms. We examined the history of placebos and the placebo effect, addressing common misconceptions and disentangling ambiguities. We then reviewed whether the placebo effect can be robustly shown in the current literature, and zoomed in on the plausible mechanisms (conditioning, expectancies and affect manipulation) through which the placebo effect might be produced. Observing the link with the doctor-patient communication literature, and pleading for a better integration of the two research traditions we conclude by setting out a research agenda for testing the role of communication in placebo effects.
\end{abstract}

\section{INTRODUCTION}

Medical history is full of anecdotes. One famous anecdote in the placebo literature is about Henry Knowles Beecher, the well known Boston anesthesiologist who worked in Europe and North-Africa during World War II. The story is about a precarious situation in a field hospital at Anzio Beachhead (Italy) where Beecher once had to perform surgery when morphine ran out while wounded soldiers kept on being brought in. In absence of morphine but unable to watch the wounded soldiers suffering, one of the nurses decided to administer a saline solution, telling the soldiers they were getting a strong pain reliever. To the amazement of all people present, this seemed to benefit the soldiers. Beecher found this such an amazing phenomenon, that after the war, he decided to make it the focus of his scientific work. In 1955 he wrote a paper in the Journal of the American Medical Association titled "The Powerful Placebo", in which he concluded, after having reviewed 15 published intervention studies, that " 35 percent of a drug's or a doctor's success is due to the patient's expectation of a desired outcome, or the placebo response" [1]. With more than 1000 citations, and despite later criticism about the soundness of the applied methodology, this paper is still one of the most cited publications in the placebo literature [2] and [3]. At the time of publication, it induced a sensation, because it demonstrated that the results of many trials were mistakenly attributed to specified interventions (usually medications), while in fact these were caused by factors outside the medical treatment itself, summarized by the term 'placebo response'. In theory, the scientific world could have reacted to this discovery in two different ways: [1] by asking the logical question: "if it is not the intervention, what else is it that heals the patient?", and studying those factors in the healing process, that apparently could not be attributed to the experimental intervention as such, or [2] by choosing a more methodological approach, in which distracting placebo effects are controlled in order to get a better view on the genuine effects of the medical intervention itself. Both approaches would have been scientifically challenging as well as clinical relevant. Standing on this crossroads, and given his fascination for the 
placebo phenomenon, Beecher could have chosen for the first approach, but, in fact, he did choose for the second one, and many clinical researchers followed in his wake. As it was, Beecher became one of the founding fathers of the RCT, the double blind, placebo-controlled Randomized Clinical Trial: the methodological answer to the discovery of placebo effects in clinical trials [3]. Soon, the RCT became the golden standard for clinical intervention studies and the firm foundation for the fast evolving paradigm of evidence-based medicine. Since then, clinical research has been walking the road of the RCTs to control for placebo effects, while at the same time avoiding to deal with them. Placebo effects are generally ignored or considered as a nuisance [4].

Still, something is gnawing. As we will show later in more detail, there is a bunch of literature showing that placebo effects are real and often substantial [5], [6], [7] and [8]. All kinds of non-specific therapeutic effects occur within the healing process, apart from the given medical treatment and the natural course of disease [9] and [10]. Moreover, throughout history, physicians have always been aware of their own healing power, even in absence of an effective medical treatment [11], [12] and [13]. Why would we ignore a phenomenon that can produce health effects? Would it not be better to study the mechanisms that might explain how these health effects are produced, apart from or on top of the medical treatment itself [14]. Or in other words: would it not be better to explore the black box of the physicians healing power [12]. With this paper, we want to show that it is worthwhile to study the plausible mechanisms behind placebo effects which might explain how health effects are actually produced. Knowing the mechanisms behind placebo effects means that it becomes possible to analyze how these mechanisms can be activated by targeted interventions. In this paper, we will focus on communication interventions, because these have been shown to be able to provide health effects and can effectively be taught in medical education and post graduate training [15], which facilitates later implementation of the results.

In summary: this paper discusses the role of communication in placebo effects. We will start with a description of what exactly is meant by the term 'placebo' and 'placebo effects', including a short historical tour and a description of the current state of the art. Then we will describe three plausible mechanisms for the production of placebo effects (conditioning, raising expectancies and reducing stress and anxiety). In this description we will show what the underlying hypothesis and the available evidence is for each of the three mechanisms, illustrated by some telling empirical examples. We will include recent knowledge from the neurocognitive sciences by showing the neurobiological substrate of each of the three proposed mechanisms. Finally we will formulate a research agenda for studying the role of communication in placebo effects by deconstructing the fuzzy concept of doctor-patient communication in elements which fit with the described mechanisms, while at the same time pleading for some methodological innovations. Hopefully, this will inspire researchers to develop a new generation of intervention studies to provide 'hard' evidence for the 'soft' side of medicine.

\section{THE AMBIGUITY OF THE 'PLACEBO'-CONCEPT}

Sometimes patients' health improves after having received medical treatment, while physicians are certain that the treatment itself did not contain any known active medical substance. This mysterious phenomenon is called 'placebo' and its effect 'placebo effect' or 'placebo response'. The medical world has always been ambivalent towards this phenomenon, not knowing whether to embrace or discard it. Physicians have done both.

Placebo is Latin for "I will please", which at first glance has a positive connotation. The pleasing aspects of the placebo concept were already reflected in the definition of 'placebo' when the concept was originally introduced in Hooper's Medical Directory in 1811: "an epithet given to any medicine given more to please than to benefit the patient" [16]. Note, however, that in this definition "pleasing" is positioned against "benefit”, suggesting an inherent discrepancy between 'pleasing' and 'benefiting' the patient. Furthermore, the definition refers to a conscious and deliberate act by the physician, Together, these characteristics have placed the placebo concept at the shady side of medicine, as was already described in the JAMA in the beginning of the previous century: "In my experience the educated physician who knows that only a few of his patients can be much benefited by drugs, gives out just as many prescriptions as the ignorant physician who believes all that the Pharmacopoeia and the nostrum vendor tell him. The only difference is that the educated physician gives his drugs as placebos. In my opinion, the placebo habit of giving drugs to every people with full faith in their pharmacologic action ... They weaken the confidence of the patient in the physician, because every placebo is a lie, and in the long run the lie is found out. We give a placebo with 
one meaning; the patient receives it with quite another. We mean him to suppose that the drug acts directly on his body, not through his mind by means of expectant attention. If the patient finds out what we are doing, he laughs at it or is rightly angry with us. I have seen both the laughter and the anger - at our expense. Placebo giving is quackery. It also fosters the nostrum evil.” [17]. This citation is not a reflection of an isolated opinion. The duality that has been ruling medicine since the times of Descartes, placing all factors beyond the biomedical domain firmly outside the domain of medical science, turned the placebo concept into an unpopular topic associated with quackery [18], or as Howard Brody puts "[many modern physicians ...] view the term "placebo" as highly stigmatized and suggestive of quackery. In their view it is non-sense to suggest that one can be both a scientific physician and a "walking placebo" [19]. This unpopular view of the placebo concept has become stronger and stronger over time, due to changing ethical standards. In short: making patients believe that a verum is given, when in fact sham medication is administered means that patients are deceived, while in modern medicine they have the right to be completely informed about the treatment, its side effects and possible alternatives. Despite these negative connotations, prescribing placebo medication (i.e. pills without a medical active substance), injecting saline, or even performing sham surgeries has remained common practice throughout medical history [3]. At a time when the biomedical sciences had little to offer (more or less till after World War II) this was quite understandable, because physicians usually had little rational alternatives to offer to their patients. It is more surprising that in the beginning of the 21st century - despite the explosion of medical knowledge, it is still common practice. Illustrating in this respect is a very recent study among American internists and rheumatologists which showed that around half of these physicians prescribe placebo treatments on a regular basis [20]. Most of the respondents in this study said they would prescribe a sugar pill for patients with a chronic painful condition if there was evidence of placebo efficacy, and nearly half said they had used treatments primarily to promote positive expectations in their patients more than once in a month, and found this ethically permissible. The major difference with previous centuries is that the modern placebo usually takes the form of a vitamin or an antibiotic, instead of a bread pill or saline injection [20]. In clinical practice, physicians try to maximize the placebo effect. Paradoxically, the "same" physicians try to minimize its effect in medical research. In other words: the placebo effect is, at the same time, a powerful therapy and obstacle to avoid when science is considered [21]. Despised but yet widely used, the placebo concept has a high level of ambiguity. No wonder the medical world is ambivalent. This makes it relevant to establish the extent to which placebo effects do indeed have an impact on patient health and to try to understand the mechanisms behind it.

\section{ARE PLACEBO EFFECTS REAL AND ROBUST?}

During the last decennium several literature reviews have been published based on narrative or systematic reviews [5], [8], [22], [23], [24], [25] and [26]. The bottom line is that most, but not all researchers argue that placebo effects are real and robust. Interestingly placebo effects are found along a range of illnesses, from medically unexplained symptoms [27] to heart failure [28] to schizophrenia [25]. Most research is focused on pain [24], [25], [29], [30] and [31], but also robustly demonstrated on Parkinson Disease [32] and [33], depression [34] and many other illnesses [25] and [35]. Stewart-Williams and Podd list and discuss a number of well designed studies which support that placebo effects are genuine on both subjective and objective parameters [7]. Vase et al. found in their meta-analysis on placebo analgesia that placebo effects on pain were indeed present, but their magnitude varied greatly. They found much higher effect sizes when a placebo condition was used to research placebo analgesia, than when a placebo condition was used only as control condition [6]. Sauro and Greenberg found in their meta-analysis that a substance administered in full view of the individual, with the suggestion that the substance would alleviate pain, induced a significant reduction in the experience of pain, whether pain was experimentally induced or created by surgical experience (effect size 0.89) [36]. Wager et al. found that placebo analgesia was related to decreased brain activity in pain-sensitive brain regions, including the thalamus, insula, and anterior cingulate cortex, and was associated with increased activity during anticipation of pain in the prefrontal cortex, providing evidence that placebos alter the experience of pain [37]. Colloca and Benedetti show that mental events induced by placebo administration can activate mechanisms that are similar to those activated by drugs, which indicates a similarity between psychosocial and pharmacodynamic effects [31]. Price et al. conclude that many placebo responses have been identified, driven by different mechanisms and are more than just response biases [25]. Not all authors report positive findings only: an impressive meta-analysis by 
Hrobjartson and Gotsche arrives at more sceptical conclusions. They found little evidence in general that placebos had powerful clinical effects. According to them placebos had no significant effects on objective or binary outcomes, they had possible small benefits in studies with continuous subjective outcomes and for the treatment of pain [22] and [23]. The critics of these critics argued in their turn that Hrobjartsson and Gotzsche's review did not find a robust placebo effect in their meta-analyses, because dichotomous outcomes can obscure actual improvement (e.g. no pain vs pain) which would be visible in continuous outcomes (e.g. a decrease in pain), because they included studies where no or a weak placebo effect can be expected (e.g. studies with neonatals) and because they lumped together studies on many patient groups with very diverse medical problems and very different study goals [26] and [38]. Indeed in their latest update, Hrobjartsson and Gotzsche [24] found, in congruence with Vase, that studies developed to research the placebo effect reported much larger placebo effects than studies merely including a placebo group as control group. It is also more likely for placebo effects to occur in clinical practice than during (some) randomized controlled trials: if patients know that they might or might not get an effective treatment, their expectations - and as a result the placebo effect - might depend on whether or not they believe to be included in the verum or in the placebo arm of a research. In sum: although there is still a considerable amount of skepticism about the nature and extent of the placebo phenomenon in part of the scientific world, there is yet sufficient evidence from the literature that placebo effects do exist, are present where you can logically expect to find them, and can be clinically important in some cases. The cited review studies on placebo and placebo effects have enough substance to invest in a better understanding of this intriguing phenomenon 4. Pleasing versus benefiting the patient: why should not we do both?

As was stated before, in the original definition, placebo was defined as "an epithet given to any medicine (or broader: any medical treatment) given more to please than to benefit the patient", suggesting an inherent discrepancy between medical interventions which please the patient and interventions that are beneficial for the patient. This has probably been one of the major reasons for the negative connotations of the placebo concept, placing it outside the realm of serious medicine. However, pleasing the patient is not by definition opposite to benefiting the patient. Throughout the history of pre-modern medicine and modern medicine, pleasing has always been acknowledged as an important part of physicians' treatments. The fact that the "pleasing" component of medical treatment might actually lead to some benefit for the patient was already noted about 24 centuries ago when Hippocrates wrote that "some patients, though conscious that their condition is perilous, recover their health simply through their contentment with the goodness of the physician" (ethics VI). It connects to what since then has been called the art of medicine: good bed-side manners, communication skills that all physicians should have. The healing potential of the art of medicine has been acknowledged ever since. In 1957, the British psychiatrist Michael Balint described the physician to be the most potent drug [11], shifting the focus from the treatment to the way the medical treatment was administered. In 1987, at the conference 'Dialogue at Wickenburg Conference, a group of highly esteemed physicians and other scientists explicitly addressed the impact of a good doctor relationship on patients' health and well-being, urging researchers to shift their focus to unraveling 'the black box of the physicians' healing power" [12]. The fact that communication skills training has become a part of the medical curriculum in medical schools all over the western world shows the general acceptance of the central role of doctor-patient communication in the delivery and effectiveness of care. There are also empirical indications that doctor-patient communication plays a role in the effectiveness of care [39], either directly, by reducing stress and anxiety, or indirectly, by influencing patient adherence, self-efficacy and satisfaction [14]. This claim was substantiated in a recent Cochrane systematic review which was cautiously optimistic about the evidence base for the effectiveness of doctor-patient communication on patient outcomes, although it also remarked that the methodological quality of many studies in this area is still suboptimal and more studies are needed [40]. Lack of progress is caused by the fact that many studies in the field of doctor-patient communication are not experimental, but observational studies, using communication as a container concept, and not identifying tailored patient outcomes, thus missing the methodological rigor to produce hard evidence [41]. Yet, current developments are promising and there is a growing literature that empirically shows that patients do benefit from the art of medicine, especially from interventions in doctorpatient communication. 


\section{THE ROLE OF DOCTOR-PATIENT COMMUNICATION IN PLACEBO EFFECT: A NATURAL TARGET FOR RESEARCH}

It might seem surprising, but, until recently, there was hardly any connection between the placebo literature and the literature on doctor-patient communication. In the course of time, two separate lines of research have emerged and found their way in separate parts of the literature. One research line (the placebo line), starts with observing health effects resulting from what is believed to be 'inactive treatment' (placebo treatment) and tries to establish the extent of these health effects and why these health effects occur. The second research line (the communication line) starts at the other end of the process, studying 'the goodness of the doctor' with an emphasis on doctor-patient communication and its impact on patient outcomes, including health effects. It is only fairly recently, that the two research lines are being brought together in an explicit attempt to establish the role of doctor-patient communication in producing placebo effects [5], [8], [9], [39] and [42]. The integration of the two research lines is promising, because it sheds light on the mechanisms that are responsible for producing health effects apart and above the medical treatment, and on the way these mechanisms can be manipulated by medical teaching and training. For instance, Di Blasi et al. studied placebo effects instigated by the patient-practitioner relationship and concluded that although there is much inconsistency, there is a relatively consistent effect for physicians' who adopt a warm, friendly, and reassuring manner: they are more effective than those who keep consultations formal and do not offer reassurance [5]. In their review of the literature, Crow et al. [8] drew attention to the important role of patient expectations in producing health effects (for instance by improving their self-efficacy or boosting their optimism on a favorable course of disease) and to physicians' possibilities to enhance or to lower these expectations [8]. The attractiveness of integrating the two research lines is that the whole chain from physician's behavior to patient outcome is under scrutiny. This makes it relevant to take a closer look at the mechanisms, which are mentioned in the literature as being responsible for producing placebo effects.

\section{MECHANISMS BEHIND PLACEBO EFFECTS}

Three mechanisms are put forward in the literature as being able to produce placebo effects: (1) conditioning, (2) the manipulation of expectancies, and (3) influencing patients' affective state and stress level. Of these three mechanisms, the manipulation of patient expectancies has received the most attention, but all mechanisms are supported by empirical evidence. Note that all three mechanisms can produce positive as well as negative outcomes. For instance, when physicians show (verbally or nonverbally) to have little confidence in the suggested treatment, this will lower patient expectancies towards a favorable treatment outcome, which can produce a negative placebo effect (nocebo-effect). Furthermore, it is important to realize that these mechanisms are interlinked in many ways and disentanglement is often difficult, except in an experimental setting. Finally, it is clear that although all three mechanisms have their source in the placebo literature, each of them is or can be influenced by physician behavior, which makes it relevant to find also inspiration in the doctor-patient communication literature.

\subsection{Conditioning}

Theoretical framework: A therapy or therapeutic procedure can produce a placebo effect on health outcome, when it is associated - consciously or unconsciously - with previous experiences. This process works by the mechanism of classical conditioning: Conditioning occurs when a neutral stimulus (e.g. a physician communicating in a warm empathic way) coincides with an effect (e.g. recovery). This neutral stimulus then becomes a conditioned stimulus, which acts as an agent for subjective or objective health improvement.

Patients usually have a medical history. Previous experiences with health care (and thus also previous health outcomes) can be either positive or negative. Via the mechanism of conditioning, these previous experiences can have an impact on medical treatment later in life. Anecdotal examples are abundant, e.g. the young woman who was treated with chemotherapy for breast cancer in a certain hospital and several years later still got nauseous when driving past that particular hospital. Goebbel provides an empirical example of the conditioning mechanism: subjects in a double blind study were conditioned in four sessions, receiving an immunosuppressive drug as an unconditioned stimulus, which was paired with a distinctively flavoured drink (the conditioned stimulus) every $12 \mathrm{~h}$. After a week all subjects were again exposed to the conditioned stimulus (drink), but now paired with placebo capsules. Although the capsules did not contain any active substance, the objectively measurable immune functions were suppressed, just like after having received the immunosuppressive drug [43]. The mechanism of conditioning has also been shown to cause 
placebo effects in, for example, Parkinson Disease and pain [7] and in the experience of side effects [35] and [44].

Conditioning cannot only produce health effects in absence of a verum treatment, the mechanism can even be responsible for an inverse reaction to treatment. Rocha do Amaral and Sabbatini [45] conditioned dogs with a loud sound that coincided with an injection with acetylcholine, lowering the dog's blood pressure. When the dogs were then exposed to the sound combined with an injection of adrenaline, which should normally heighten dogs' blood pressure, the blood pressure dropped instead. Translated to a human situation this phenomenon could explain why patients do not react or even inversely react on certain medication. This means that physician when confronted with unusual reactions to medical treatment should always ask about and take into account the patient's previous experiences with treatment. It is important to note that patient expectancies can be in line with a conditional stimulus-response pair, put also in reversed direction. In such cases, expectancies might be strong enough to reverse the conditioning effect [46]. This means that when physicians have become aware of conditioning processes in the patient with negative impact on health outcomes, it might be possible to diminish these effects by influencing patient expectations, Conditioning is often, but not always an automatic unconscious process depending on previous experiences. However, sometimes patients are well aware of their previous experiences. In these cases, the mechanism of conditioning is closely interlinked with the mechanism of expectancies.

\subsection{Expectancies}

Theoretical framework: A medical treatment or procedure can produce a placebo effect because the recipient of the treatment expects it to. The expectancy-mechanism differs from conditioning as it is always a conscious process. Expectancies help to prepare body and mind for what is going to come. Expectancies may be seen as either the patients' beliefs about the effects of treatment (outcome expectancies) and the patients' beliefs about their abilities to carry out or cope with the disease and its treatment (self-efficacy) [7] and [8] The most studied mechanism in placebo research is the impact of patients' positive or negative expectancies. An extremely lucid design to show the power of expectancies is the open/hidden paradigm. In such designs medication is administered either by an automatic pump (i.e. patients are unaware when the medication starts or stops) or by a nurse who explains when and what kind of medication is being administered. With several types of painkillers, patients experience more and faster pain relief if they are aware that the analgesic is administrated [25], [29], [31] and [32], a similar effect was found for benzodiazepines. Also, when patients were aware that their pain medication was no longer administered, they immediately felt an increase in pain. When patient were unaware of no longer receiving the medication, their pain relief continued much longer [32]. This approach also highlights that a placebo effect is also able to complement (or detriment in case of nocebo responses) real, active treatments.

Much progress in knowledge on how expectancies can lead to placebo effects is made thanks to the application of the neurocognitive sciences in pain research and through the use of modern imaging techniques, such as fMRI and PET-scans. Levine and Gordon first discovered the role of the body's endogenous opioid system in producing placebo analgesia [47]. Expectancy-induced release of endogenous opioids has been obtained by using in vivo receptor binding with positron emission tomography [48]. Similar regions in the cerebral cortex and in the brainstem are affected by both a placebo and the painkiller remifentanil [48].

Expectations thus often play an important role in producing placebo effects, These effects can be influenced both by previous experiences and/or verbal suggestion. It seems that expectations mimic the effects of opioid drugs. Amazingly, this is not just a general effect on the whole body. Using fMRI-scans, Lieberman et al. were able to show in subjects who were treated with a placebo cream on one hand but not on the other, that brain activation associated with pain was markedly different in areas associated with the 'treated' arm versus the untreated hand or foot [49].

We conclude that there are many studies showing the influence of expectancies on health outcomes. Clear neurobiological substrates of this mechanism and its effects can be discerned. These effects are genuine, not only response bias. Additionally, expectancy related placebo effects are larger when patients have a stronger desire to reach a positive goal or when avoiding an unpleasant situation [25] and when patients are focused on their somatic symptoms [50]. The role of physician communication in raising, lowering or directing patient expectancies is clear. However, communication is a complex process, and is too often used as container concept, blurring the view on the effective elements [41]. Lacking in the literature are studies 
that clearly make a distinction between raising positive or negative expectations and positive affect, e.g. a friendly and empathic doctor.

\subsection{Affect manipulation}

Theoretical framework: Patients' affective states are influenced by the way a treatment is administered. Being ill, the fear of being ill, but also the medical visit itself is for many people a stressful event. Distress resulting from illness might decrease, for instance because a physician provides empathy and support, or increase, when a patient feels misunderstood, is treated in a cold unfriendly manner or receives bad news. The resulting change in affect, the conscious subjective aspect of feeling, can lead to placebo effects in various ways.

Different theories propose a path through which affect can impact health or treatment outcomes, for instance by stress reduction: in human studies it was demonstrated that the down-regulation of the early inflammatory response by an increase in cortisol levels resulted in delayed wound repair. Moreover, in this study several potential cellular mechanisms linking stress and wound healing were identified [51]. In pain patients, symphathetic activation by a sudden stressor can increase pain [52]. Social support might decrease pain [53], and on the long term lead to a better health [54].

In patients with pain, low to intermediate levels of negative affect can facilitate pain in uncontrollable situations [55], this might work by shifting attention and amplificating pain through pain and stress related neural circuitry like the amgygdala (the processing of negative emotions shares neural networks with pain responses [56]). Negative affect might also lead to hypersensitivity to pain, possibly through heightened concentration of catecholomines (fight-or-flight hormones) in response to psychological stress [57], by misattribution of arousal or selective attention [58]. Other clues pointing to the role affect in are the heightened placebo response in patients that are anxious or stressed [59]. Also, placebo effects are usually greater in clinical pain, where affect arguable plays a larger role than in experimental pain [25]. Furthermore, desire for relief, which can be defined as both a motivational and affective state is an important factor apart from expectancies [25] and [46].

Apart from direct influences of affect manipulation on health outcomes, affect can also work through moderators like self-disclosure, adherence and self-efficacy [5], [25] and [60]. The role of affect is especially evident in studies on the effects of doctor-patient communication, where there is traditionally a focus on the importance of providing empathy and communicating in a warm and friendly fashion. Affective communication (warmth, empathy, eye contact) has shown to be related to several patient outcomes, including satisfaction, adherence and health outcomes (Hb1ac, blood pressure) [5], [61], [62] and [63]. It is likely that there is an interaction of affect with expectancies: positive or negative expectancies might lead accordingly to more positive or negative affect, while a change in affect might lead to according changes in expectancies by interpreting situations more positively or more negatively. In the placebo literature these refined distinction between different elements of physician communication is not yet made.

\section{SOME FINAL REFLECTIONS AND A RESEARCH AGENDA}

Placebo effects exist and are probably substantial in many cases. However, studying placebo effects is not very popular in medical science: most clinical researchers choose to control for placebo effects in double blind, placebo-controlled randomized clinical trials, rather than making the observed placebo effects the focus of their research efforts. Placebo effects are unpopular in clinical science for several reasons. First, because by definition, placebo effects are placed outside the scientific realm. Second, because they blur the view on the genuine efficacy of medical treatment, which hampers the progress of medical knowledge. Fourth, and related to the previous point, because the awareness of unexpected placebo effects produces negative feelings in its practitioners when a trusted treatment gets unmasked by a new series of clinical trials. Throughout history, therapeutic effects are often misattributed to a new type of treatment, while in fact only placebo effects were in effect. With hindsight, an alarming amount of therapies which were commonly administered at a certain point in time, have later been shown to be just another example of placebo treatments [64]. This demonstrates that physicians are not always aware that they administer placebo treatments. Roberts even concluded that placebo effects are strongest when both physician and patient believe in the efficacy of the administered treatment. Physicians may feel fooled when empirical evidence shows the superior impact of placebo effects over the effects of the intended medical treatment. This does not contribute to the popularity of the placebo concept. Fifth, and probably most important, not only physicians might feel fooled, but also patients are deceived by placebo treatment, and might react 
accordingly. By contemporary ethical standards, patients have the right to be fully informed about the proposed medical treatment, its side effects and possible alternatives. This is at odds with the conscious prescription of placebo treatment. Yet, the prescription of placebo treatment is still common, as was shown in a recent paper in BMJ [20], while the unconscious use of placebo treatments has to be huge, considering the continuously changing content of clinical guidelines and medical handbooks. No wonder that clinical researchers want to get rid of those nasty placebo effects. When analyzing the reasons for the unpopularity of the placebo concept, Beecher's choice, to focus on developing a methodology to control for placebo effects in medical intervention studies, rather than analyzing the healing ingredients of this mysterious concept, can easily be understood. Placebos still dwell at the shady side of medicine.

This might change when we approach the same topic from a different angle: the angle of doctor-patient communication. Few physicians will deny the important role of doctor-patient communication in the development of a trustful doctor-patient relationship as well as in the diagnostic and therapeutic process. For many physicians, this is a deep personal experience. There is also some empirical evidence on the therapeutic value of patient centred communication which might lead to positive health outcomes, although the methodological quality of many studies in this field is moderate and more research is needed [40].

This is exactly where both research traditions can strengthen each other to the benefit of both. Placebo research could learn from the communication literature to fine-tune its experimental interventions in a way to appeal to physician's clinical needs instead of producing feelings of guilt, shame and powerlessness. This would enhance the clinical relevance of placebo research, and probably pull this line of research out of the shadows, and put it right into the clinical arena, i.e. the medical consultation room, the hospital ward or the nursing home The open-hidden research paradigm that was described as a way of experimentally manipulating patient expectancies is a good example of a clinical relevant placebo study.

On the other hand: researchers in doctor-patient communication can learn a lot from placebo effect researchers: the methodological rigor of their experiments, the application of the rapidly developing knowledge from the neurocognitive sciences, and the use of modern imaging techniques for establishing physiological evidence for the assumed mechanisms in placebo effects as a result of narrowly defined interventions.

On paper, this could be a happy marriage. But in order to develop a sustainable relationship both parties have to be aware of the different cultures in the two research traditions. It will be no coincidence that there has been hardly any integration of the two fields till now. Placebo researchers love to work under strictly controlled laboratorium-like conditions; inspired by the lab, the clinic is often far away, even when working with patients. Communication researchers on the other hand tend to adopt a holistic view on medicine and to use communication as a container concept which cannot be dissected in separate elements. This dissection is needed to put elements to test under strict experimental conditions. Inspired by the clinic, the lab is often far away, even when performing experimental intervention studies.

The challenge is clear. It is time to develop a common research agenda which unites the strong points of both research traditions. This means that the mechanisms which have proven to be active in producing health effects in placebo research ought to be analyzed on the type of communication elements which could possibly be held responsible for producing these effects. This implies the deconstruction of communication in meaningful elements, which are then systematically tested under controlled conditions. An example is given in the description of the inter-linkage of the three mechanisms: it was demonstrated that patients experience faster pain relief when the drug is administered by a nurse who assures the patient that the drug is highly effective as compared to a situation where the drug is administered by a computerized pump. However, what is responsible for the results: the raising of positive expectations or the stress-reducing empathic attitude of the nurse? By systematic variations in the experimental design the separate and combined effects of various communication elements can be tested on the outcomes they produce on the patient.

Often, for practical as well as ethical reasons, it will not be possible to perform this type of experiments on real patients. Moreover, because of its interactive characteristics, it is difficult to fully standardize communication in clinical situations. A solution for this problem is: to develop video vignettes in which communication elements are systematically varied according to a protocol, and showing these vignettes to analogue, instead of real patients. The use of analogue patients is common in doctor-patient communication research, although little attention has been paid to the validity of this approach. However, the discovery of the mirror neuron system in humans [65], [66], [67], [68], [69] and [70] which is responsible for the 
empathic competence of humans to put themselves in the shoes of suffering others, make it plausible that letting subjects watch a video of a medical consultation is a promising new type of methodology, which enable the study the effects of systematic small variations in physician communication, although validity checks are still needed, and new methodological questions will certainly arise. But let us try to see these as a challenge, instead of insurmountable obstacles. It is worthwhile to develop a new generation of intervention studies, because the possibility to unravel the mystery behind the Silent Healer by systematic testing of plausible connections in rigorous study designs is an inviting perspective.

\section{CONFLICT OF INTEREST STATEMENT}

The authors have no conflict of interest.

\section{REFERENCES}

[1] Beecher HK. The powerful placebo. J Amer Med Assoc 1955;159:1602-6.

[2] Kienle GS, Kiene H. The powerful placebo effect: fact or fiction? J Clin Epidemiol 1997;50:1311-8.

[3] de Craen AJ, Kaptchuk TJ, Tijssen JG, Kleijnen J. Placebos and placebo effects in medicine: historical overview. J R Soc Med 1999;92:511-5.

[4] Wickramasekera I. A conditioned response model of the placebo effect predictions from the model. Biofeedback Self Regul 1980;5:5-18.

[5] Di Blasi Z, Harkness E, Ernst E, Georgiou A, Kleijnen J. Influence of context effects on health outcomes: a systematic review. Lancet 2001;357:757-62.

[6] Vase L, Riley JL, Price DD. A comparison of placebo effects in clinical analgesic trials versus studies of placebo analgesia. Pain 2002;99:443-52.

[7] Stewart-Williams S, Podd J. The placebo effect: dissolving the expectancy versus conditioning debate. Psychol Bull 2004;130:324-40.

[8] Crow R, Gage H, Hampson S, Hart J, Kimber A, Thomas H. The role of expectancies in the placebo effect and their use in the delivery of health care: a systematic review. Health Technol Assess 1999;3:196.

[9] Dulmen AMv, Bensing JM. The effect of context in health care: a programming study; 2001.

[10] Roter D, Hall JA. Doctors talking with patients/patients talking with doctors: improving communication in medical visits. Westport, CT: Praeger; 2006.

[11] B. alint M. The doctor, his patient and the illness. London: Pitman Medical; 1957 [12] White K, editor. The task of medicine: dialogue at Wickenburg. 1988. Menlo Park.

[13] Cassell EJ. The nature of suffering and the goals of medicine. Oxford: Oxford University Press; 2004.

[14] Street Jr RL, Makoul G, Arora NK, Epstein RM. How does communication heal? Pathways linking clinician-patient communication to health outcomes. Patient Educ Couns 2009;74:295-301.

[15] Aspegren K. BEME guide no. 2: teaching and learning communication skills in medicine-a review. Med Teach 1999;21:563.

[16] Quincy J. Quincy's Lexicon-Medicum. A new medical dictionary. Revised.

London: R. Hooper; 1811.

[17] Cabot RC. The physician's responsibility for the nostrum evil. J Amer Med Assoc 1906;47:982-3.

[18] Wall PD. The placebo effect: an unpopular topic. Pain 1992;1:1-3.

[19] Brody $\mathrm{H}$. The doctor as therapeutic agent: a placebo effect research agenda.

The placebo effect: an interdisciplinary exploration. Cambridge, MA: Harvard University Press; 1997. p. 260.

[20] Tilburt JC, Emanuel EJ, Kaptchuk TJ, Curlin FA, Miller FG. Prescribing "placebo treatments": results of national survey of US internists and rheumatologists.

Brit Med J 2008;337:a1938.

[21] Macedo A, Farre M, Banos JE. Placebo effect and placebos: what are we talking about? Some conceptual and historical considerations. Eur J Clin Pharmacol 2003;59:337-42.

[22] Hrobjartsson A, Gotzsche PC. Is the placebo powerless? An analysis of clinical trials comparing placebo with no treatment. N Engl J Med 2001;344:1594-602.

[23] Hrobjartsson A, Gotzsche PC. Placebo interventions for all clinical conditions. Cochrane Database Syst Rev 2004;3:CD003974.

[24] Hrobjartsson A, Gotzsche PC. Placebo interventions for all clinical conditions.

Cochrane Database Syst Rev 2010;1:CD003974.

[25] Price DD, Finniss DG, Benedetti F. A comprehensive review of the placebo effect: recent advances andcurrent thought.Annu Rev Psychol 2008;59:565-90. 
[26] Wampold BE, Imel ZE, Minami T. The story of placebo effects in medicine: evidence in context. J Clin Psychol 2007;63:379-90. discussion 405-8.

[27] Cho HJ, Hotopf M, Wessely S. The placebo response in the treatment of chronic fatigue syndrome: a systematic review and meta-analysis. Psychosom Med 2005;67:301-13.

[28] Olshansky B. Placebo and nocebo in cardiovascular health: implications for healthcare, research, and the doctor-patient relationship. J Am Coll Cardiol 2007;49:415-21.

[29] Benedetti F, Lanotte M, Lopiano L, Colloca L. When words are painful: unraveling the mechanisms of the nocebo effect. Neuroscience 2007;147:260-71.

[30] Kong J, Kaptchuk TJ, Polich G, Kirsch I, Gollub RL. Placebo analgesia: findings from brain imaging studies and emerging hypotheses. Rev Neurosci 2007;18: 173-90.

[31] Colloca L, Benedetti F. Placebos and painkillers: is mind as real as matter? Nat Rev Neurosci 2005;6:545-52.

[32] Colloca L, Lopiano L, Lanotte M, Benedetti F. Overt versus covert treatment for pain, anxiety, and Parkinson's disease. Lancet Neurol 2004;3:679-84.

[33] de la Fuente-Fernandez R, Schulzer M, Stoessl AJ. Placebo mechanisms and reward circuitry: clues from Parkinson's disease. Biol Psychiatry 2004;56: 67-71.

[34] Dworkin RH, Katz J, Gitlin MJ. Placebo response in clinical trials of depression and its implications for research on chronic neuropathic pain. Neurology 2005;65(Suppl 4):S7-19.

[35] Barsky AJ, Saintfort R, Rogers MP, Borus JF. Nonspecific medication side effects and the nocebo phenomenon. J Amer Med Assoc 2002;287:622-7.

[36] Sauro MD, Greenberg RP. Endogenous opiates and the placebo effect: a metaanalytic review. J Psychosom Res 2005;58:115-20.

[37] Wager TD, Rilling JK, Smith EE, Sokolik A, Casey KL, Davidson RJ, et al. Placeboinduced changes in FMRI in the anticipation and experience of pain. Science 2004;303:1162-7.

[38] Wampold BE, Imel ZE, Minami T. The placebo effect: "relatively large" and "robust" enough to survive another assault. J Clin Psychol 2007;63:401-3.

discussion 405-8.

[39] Dulmen AMv, Bensing JM. Health promoting effects of the physician-patient encounter. Psychol Health Med 2002;7:289-300.

[40] Lewin SA, Skea ZC, Entwistle V, Zwarenstein M, Dick J. Interventions for providers to promote a patient-centred approach in clinical consultations.

Cochrane Database Syst Rev 2001;4:CD003267 [updated in 2010].

[41] de Haes H, Bensing J. Endpoints in medical communication research, proposing a framework of functions and outcomes. Patient Educ Couns 2009;74:287-94.

[42] Hrobjartsson A. What are the main methodological problems in the estimation of placebo effects? J

Clin Epidemiol 2002;55:430-5.

[43] Goebel MU, Trebst AE, Steiner J, Xie YF, Exton MS, Frede S, et al. Behavioral conditioning of immunosuppression is possible in humans. FASEB J 2002; 16:1869-73.

[44] Benedetti F, Amanzio M, Baldi S, Casadio C, Maggi G. Inducing placebo respiratory depressant responses in humans via opioid receptors. Eur J Neurosci 1999;11:625-31.

[45] Rocha do Amaral J, Sabbatini RME. Placebo effect: the power of the sugar pill; 1999. Available at: http://www.cerebromente.org.br/n09/mente/ placebo1_i.htm [accessed 2010].

[46] Harrington A. The placebo effect: an interdisciplinary exploration. Cambridge, MA: Harvard University Press; 1997.

[47] Levine JD, Gordon NC, Fields HL. The mechanism of placebo analgesia. Lancet 1978;2:654-7.

[48] Zubieta J, Bueller JA, Jackson LR, Scott DJ, Xu Y, Koeppe RA, et al. Placebo effects mediated by endogenous opioid activity on \{micro\}-opioid receptors. J Neurosci 2005;25:7754-62.

[49] Benedetti F, Arduino C, Amanzio M. Somatotopic activation of opioid systems by target-directed expectations of analgesia. J Neurosci 1999;19:3639-48.

[50] Villemure C, Bushnell MC. Cognitive modulation of pain: how do attention and emotion influence pain processing? Pain 2002;95:195-9.

[51] Vileikyte L. Stress and wound healing. Clin Dermatol 2007;25:49-55.

[52] Drummond PD, Finch PM, Skipworth S, Blockey P. Pain increases during sympathetic arousal in patients with complex regional pain syndrome. Neurology 2001;57:1296-303.

[53] Brown JL. Social support and experimental pain. Psychosom Med 2003; 65:276-83.

[54] Cacioppo JT, Hawkley LC. Social isolation and health, with an emphasis on underlying mechanisms. Perspect Biol Med 2003;46(3 Suppl):S39-52.

[55] Rhudy JL, Meagher MW. The role of emotion in pain modulation. Curr Opin Psychiatry 2001;14:241-5.

[56] Eisenberger NI, Lieberman MD. Why rejection hurts: a common neural alarm systemfor physical and social pain. Trends Cogn Sci (Regul Ed) 2004;8:294-300. 
[57] Janssen SA, Arntz A, Bouts S. Anxiety and pain: epinephrine-induced hyperalgesia and attentional influences. Pain 1998;76:309-16.

[58] Janssen SA. Negative affect and sensitization to pain. Scand J Psychol 2002;43:131-7.

[59] Wasan AD, Kaptchuk TJ, Davar G, Jamison RN. The association between psychopathology and placebo analgesia in patients with discogenic low back pain. Pain Med 2006;7:217-28.

[60] Epstein RM, Street RLJ. Patient-centered communication in cancer care: promoting healing and reducing suffering; 2007, NIH Publication No. 07-6225.

[61] Stewart MA. Effective physician-patient communication and health outcomes: a review. Can Med Assoc J 1995;152:1423-33.

[62] Griffin SJ, Kinmonth AL, Veltmn MWM, Gillard S, Grant J, Steward M. Effect on health-related outcomes of interventions to alter the interaction between patients and practitioners: a systematic review of trials. Ann Fam Med 2004;2:595-608.

[63] Rao JK, Anderson LA, Inui TS, Frankel RM. Communication interventions make a difference in conversations between physicians and patients: a systematic review of the evidence. Med Care 2007;45:340-9.

[64] Roberts AH, Kewman DG, Mercier L, Hovell M. The power of nonspecific effects in healing: implications for psychosocial and biological treatments. Clin Psychol Rev 1993;13:375-91.

[65] Gallese V, Goldman A. Mirror neurons and the simulation theory of mindreading.

Trends Cogn Sci 1998;2:493-501.

[66] Rizzolatti G, Craighero L. The mirror-neuron system. Annu Rev Neurosci 2004;27:169-92.

[67] Gallese V. Before and below 'theory of mind': embodied simulation and the neural correlates of social cognition. Philos Trans R Soc Lond B Biol Sci 2007;362:659-69.

[68] Lamm C, Batson CD, Decety J. The neural substrate of human empathy: effects of perspective-taking and cognitive appraisal. J Cogn Neurosci 2007;19:42-58.

[69] Decety J, Jackson PL. The functional architecture of human empathy. Behav Cogn Neurosci Rev 2004;3:71-100.

[70] Ruby P, Decety J. How would you feel versus how do you think she would feel? A neuroimaging study of perspective-taking with social emotions. J Cogn Neurosci 2004;16:988-99. 